\title{
Pulsed-Laser Testing Methodology for Single Event Transients in Linear Devices
}

\author{
Stephen Buchner, Member, IEEE, James Howard, Jr, Senior Member, IEEE, Christian Poivey, Member, IEEE, \\ Dale McMorrow, and Ron Pease, Senior Member, IEEE
}

\begin{abstract}
A methodology for testing linear devices for single event transients that uses a pulsed laser to supplement a heavy-ion accelerator is proposed. The method is based on an analysis of plots of transient amplitudes versus width over a range of laser pulse input energies and heavy-ion LETs. Additional data illustrating the method are presented that include the dependence of SETs on circuit configuration in a comparator (LM111) and operational amplifier (LM124). If judiciously used, the methodology has the advantage that the amount of heavy-ion accelerator testing can be reduced.
\end{abstract}

Index Terms-Linear devices, pulsed-laser testing, radiation, single event transients.

\section{INTRODUCTION}

$\mathbf{L}$ INEAR circuits produce single event transients (SETs) when exposed to ionizing particle radiation in space. Such transients can propagate into follow-on circuitry and be latched as single-event upsets (SEUs). Before they can be used in spacecraft electronic systems, their SET sensitivity must be evaluated at a heavy-ion accelerator facility. This is a costly and time consuming undertaking requiring long lead times for design validation.

Linear circuits are unique in that they may be used in an almost unlimited number of configurations (e.g., supply voltage, input voltage, output impedance and gain). Previous testing has revealed that SET characteristics (pulse-shape and cross-section) in certain linear circuits depend on configuration [1]-[6]. Consequently, the SET test results for a specific configuration of a given part do not necessarily apply to different configurations of that part. Performing radiation testing on a large number of different configurations places a tremendous financial burden on the mission, and any method that reduces the amount of accelerator testing merits consideration. It is our contention that, if judiciously applied, a pulsed picosecond laser can be used to reduce the amount of accelerator testing.

Manuscript received July 21, 2004. This work was supported in part by the Defense Threat Reduction Agency and the NASA Electronic Parts and Packaging Program.

S. Buchner is with the QSS Group Inc, Greenbelt, MD 20772 USA (e-mail: sbuchner@pop500.gsfc.nasa.gov).

J. Howard is with Jackson \& Tull, Greenbelt, MD 20771 USA (e-mail: jihoward@pop500.gsfc.nasa.gov).

C. Poivey is with SGT Inc, Greenbelt, MD 20770 USA (e-mail: cpoivey@pop500.gsfc.nasa.gov).

D. McMorrow is with the Naval Research Laboratory, Washington DC 20375 USA (e-mail: mcmorrow@ccs.nrl.navy.mil).

R. Pease is with RLP Research, Albuquerque, NM 87122 USA (e-mail: rpease@mrcmicroe.com).

Digital Object Identifier 10.1109/TNS.2004.839263
Numerous reports in the literature describe the application of a pulsed laser to the evaluation of SETs in linear circuits [7]-[9], [12] These reports demonstrate the excellent agreement between SET pulse shapes generated by laser light and by heavy ions. For two particular linear devices-a voltage comparator (LM111) and an operational amplifier (LM124) — the shapes of all SETs generated by heavy ions in a variety of different configurations could be matched exactly with SETs generated by a pulsed laser [7], [12] Analogous results have been observed for a range of other devices (LM119, LM139, OP27, LM6144), but they have not been studied as extensively. Metal coverage, always an issue for pulsed-laser SEE testing, has, for the most part, not limited the acquisition of SETs in linear circuits because most transistors are relatively large and free of metal. In those cases where junctions are covered with metal, it is still possible to produce SETs by focusing the light on areas adjacent to the metal and relying on charge diffusion [7]. Using this approach, it was possible to generate the full spectrum of SETs in all linear devices tested to date.

The current maturity of the pulsed laser technique suggests that it can be part of a standard approach for characterizing the SET sensitivity of linear devices. The approach we are proposing uses the pulsed laser to reduce-but not eliminate - the amount of heavy-ion testing required to characterize SETs in linear circuits. The approach is based on analyzing plots of amplitude $(\Delta V)$ versus width $(\Delta t)$ for all SETs generated by pulsed laser light and heavy ions [10]. Once a part has been fully characterized with heavy ions for one configuration, the pulsed laser may be used to characterize it for other configurations. Furthermore, in those cases where there is no heavy-ion data, the pulsed-laser data may be used to assess whether SETs might pose a problem to a system based on its bandwidth for propagation. In both cases, the amount of heavy-ion testing is reduced.

\section{BACKGROUND}

\section{A. Previous Work}

A test methodology for evaluating SETs in linear circuits has previously been proposed [11]. The authors suggest two alternate approaches, one involving modeling and the other pulsed laser testing. At the present time, except in those few cases where validated circuit models with all the SPICE parameters are available, a modeling effort can be more time-consuming and costly than accelerator testing, particularly for COTS devices for which manufacturers rarely divulge proprietary circuit parameters. The real strength of the modeling approach is in helping to understand anomalous behavior. 
Those authors [11] also propose that SET testing be performed with the device included in the actual system, and only those SETs that propagate through the system need be considered. The problems with that approach are that: 1) devices are identified well before an actual system is built, and it is not practical to wait that long before testing is done, and 2) often the same device is used in many applications within a system or spacecraft and this would require extensive testing.

\section{B. Overview of the New Approach}

The methodology we propose involves using both heavy ions and a pulsed laser to characterize SET sensitivity of linear circuits.

Characterizing the part with heavy ions involves capturing all the transients for each ion LET using a storage oscilloscope. In order to capture all the transients, the oscilloscope's trigger level must be set very close to the dc output level. The stored waveforms are subsequently analyzed to extract values of $\Delta V$ and $\Delta t$. That information is then used to produce plots of $\Delta V$ versus $\Delta t$ for all the SETs captured with the goal of producing a plot of $\Delta V$ versus $\Delta t$ for each value of ion LET.

The next step is to characterize the part using pulsed laser light for the same configuration used to obtain the heavy-ion data. This involves focusing the laser light on each SET-sensitive transistor and capturing the SETs generated while gradually increasing the laser pulse energy. As in the heavy-ion case described above, the transient data are analyzed to generate plots of $\Delta V$ versus $\Delta t$, except that in this case the identity of the transistor responsible for each transient is known. For the laser case, all the SET data are contained in a single plot.

Should the device so characterized be needed in a different configuration, the above data, together with additional pulsed laser data obtained for the new configuration, may be used. If the bandwidth of the follow-on circuit in the new configuration is such that some of the largest transients generated in the device can propagate, rough estimates of threshold LET and cross-section may be determined.

The laser data may, by itself, be used to determine whether a potential device is suitable for use in a particular application. The criterion used for this determination is whether transients are able to propagate through the follow-on circuit. The maximum amplitudes and widths of the transients may be measured with the pulsed laser and compared with the minimum values necessary for propagation. If the maximum transients are both too small and too narrow to propagate in the follow-on circuitry, the part may be used without further testing being necessary. If the largest transients will propagate, a first option of adding filters to block propagation could be investigated. The bandwidth requirement for those filters can be determined from the maximum values of $\Delta V$ and $\Delta t$ measured in the laser experiment. In this way additional heavy ion testing may be avoided.

\section{Analysis Using $\Delta V-\Delta t$ Plots}

Fig. 1 shows an example of a $\Delta V-\Delta t$ plot obtained for the LM124 operational amplifier in one particular configuration (voltage follower, $V_{\mathrm{dd}}=15 \mathrm{~V}, V_{\mathrm{cc}}=-15 \mathrm{~V}$ and $V_{\mathrm{in}}=5 \mathrm{~V}$ ) [12]. Data collection involved the use of pulsed laser light with a wavelength of $590 \mathrm{~nm}$ that gave a penetration (1/e) depth

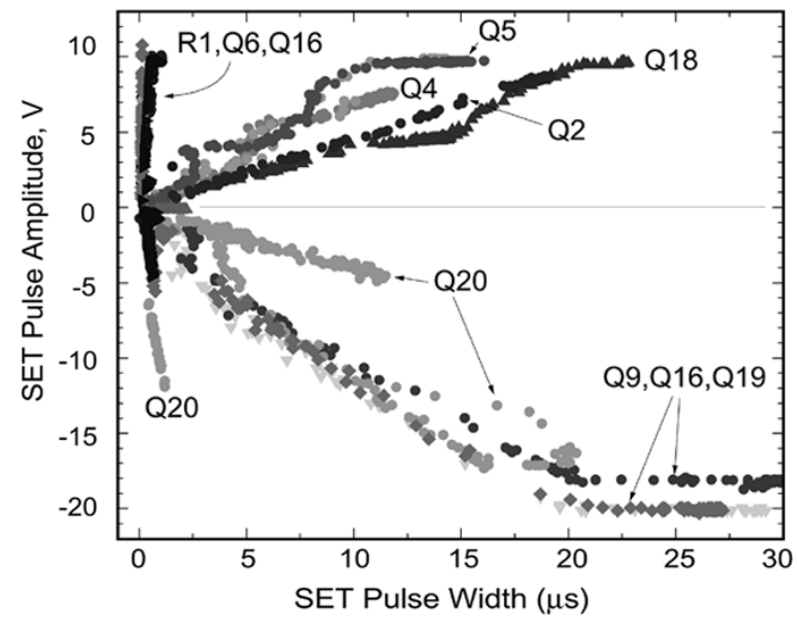

Fig. 1. Laser-induced SET amplitude versus pulse width for SETs generated in the LM124 operational amplifier [12].

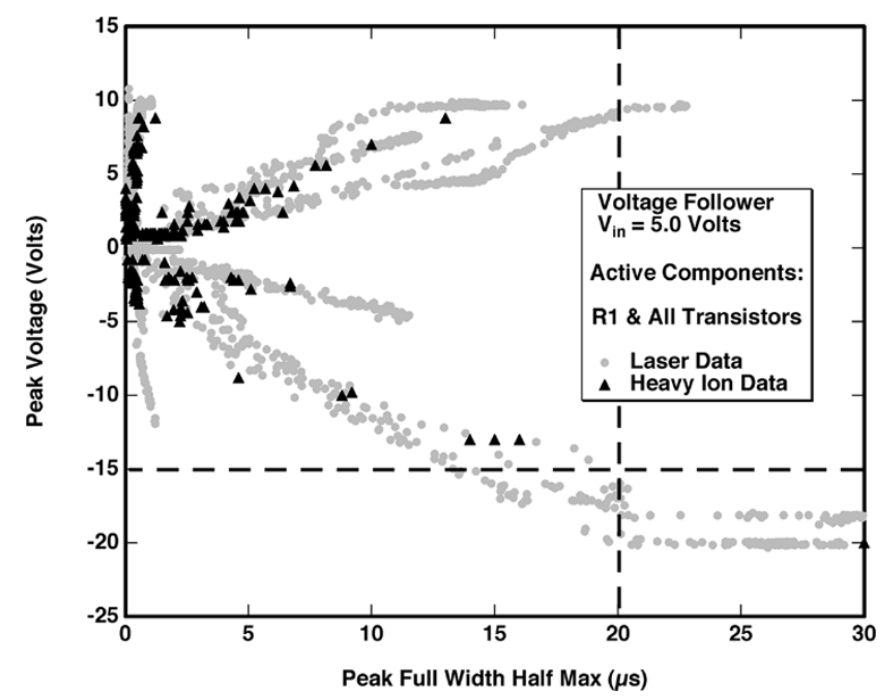

Fig. 2. Plot of laser-induced $\Delta V$ versus $\Delta t$ illustrating the "phase space" $(15 \mathrm{~V}>\Delta V>-15 \mathrm{~V}$ and $20 \mu \mathrm{s}>\Delta t)$ where SETs do not propagate. Clearly, the ones that will propagate for this configuration are in the lower right-hand quadrant and originate in one or more of the transistors Q19, Q16, and Q9 [12].

of $2 \mu \mathrm{m}$. The light was focused to a spot with a diameter of $1 \mu \mathrm{m}$ on one transistor at a time. An oscilloscope together with a low-capacitance $(11 \mathrm{pF})$ probe connected directly between the device output and the oscilloscope were used to capture all SETs with amplitudes greater than $0.5 \mathrm{~V}$. For each node, SETs were continuously captured as the laser pulse energy was gradually increased. By repeating this procedure for all SET-sensitive transistors, a complete SET spectrum is obtained. A computer program is used to extract values of $\Delta V$ and $\Delta t$ for each SET. The results are then plotted as shown. By referring to a circuit diagram, the identity of the transistor responsible for each $(\Delta V, \Delta t)$ point may be established [12].

SETs induced by heavy ions $\left(\right.$ LET $=59 \mathrm{MeV} \bullet \mathrm{cm}^{2} / \mathrm{mg}$ ) were also captured and analyzed for the same device under identical operating conditions. Using the same data analysis program, the values of $\Delta V$ and $\Delta t$ for all SETs were extracted and the resulting data overlaid on the pulsed laser data, as illustrated in Fig. 2. Clearly, the ion data are coincident with the pulsed-laser data. This result is not unexpected considering 
the excellent agreement previously observed between individual SET pulse shapes generated for this device using heavy ions and pulsed laser light [7], [9]. Another advantage of this approach is the ability to identify the origins of each one of the ion data points, something that is normally not possible when using a broad ion beam. Producing this plot for low LET ions allows one to determine unambiguously which transistors are the most SET sensitive. We note that the data are for a particular configuration, and the question remains as to how the distribution of data points changes with supply voltage, input voltage and device configuration.

One point previously mentioned is that not all SETs generated in the linear device will propagate through the system as a whole. Some pulses are either too small or too narrow to propagate. Knowledge of the minimum pulse width and amplitude required for SET propagation through a particular system allows for the definition of a region in $\Delta V-\Delta t$ space where SETs will not affect the follow-on circuitry and can be ignored. This is illustrated in Fig. 2, where it is assumed that all SETs with amplitudes smaller than $\pm 15 \mathrm{~V}$ and pulse widths shorter than $20 \mu$ s will not propagate through the follow-on circuitry. Such information will, in general, be supplied by the design engineer and will vary from application to application. The values chosen in this example are for illustrative purposes only. The area in the upper left quadrant of Fig. 2 delineates the phase space for which propagation through the follow-on circuit will not occur. For this example it is necessary to consider only those SETs that fall within the box at the bottom right-hand corner, because those are the only ones that will propagate through the system. All other SETs may be ignored.

Figs. 1 and 2 together show clearly that, for this example, transistors Q9, Q16, and Q19 are the only ones capable of producing SETs that will propagate. If the device is to be used in the same configuration but with different follow-on circuitry, the limits of $\Delta V$ and $\Delta t$ on the graph are moved accordingly. The graph is then inspected to ascertain whether there are any SETs that will propagate.

The ion data in Fig. 2 are for ions with the highest LETs used during the run. The pulsed laser is able to deposit more energy than the accelerator ions merely by increasing the pulse intensity. Therefore, it is not surprising that the laser can produce SETs that are larger and longer than those produced by heavy ions. The only limits on pulse amplitude and width are imposed by the circuit response and by thermal damage due to absorption of very high laser light intensities in the silicon.

\section{Test Methodology}

Fig. 3 shows the steps involved in applying this methodology to help qualify a linear part for its SET sensitivity in an ionizing particle environment. Note that the final step always involves an optional accelerator test, except in the case where ion data are already available for the particular configuration being considered. Inspection of all the steps involved suggests that judicious use of a pulsed laser can significantly reduce the amount of heavy ion testing needed to qualify a linear device for SET sensitivity.

The first step in the test methodology is to determine whether any SET data already exist for the configuration of interest. Plots of cross-section as a function of ion LET are necessary but not sufficient. The data should also include complete waveforms for all the captured SETs for each value of ion LET. That data is then used to generate plots of $\Delta V$ versus $\Delta t$. A computer program has been developed to rapidly analyze all the transients (from very large data sets) and produce plots of $\Delta V$ versus $\Delta t$, analogous to those in Figs. 1 and 2. An additional useful feature of the program is the ability to cull from the data plots of $\Delta V$ versus $\Delta t$ for a single transistor under a wide variety of operating conditions. It is then simple to see how the transistor's response depends on operating condition.

In order to establish the minimum acceptable values of amplitude and width, it is necessary to consult a design engineer to determine the conditions under which SETs will propagate through the system. At this point, two different paths are followed, depending on whether or not SET data are already available. The two paths are discussed in more detail in Sections IV and $\mathrm{V}$.

\section{A. No Heavy-Ion SET Data Available}

The situation is relatively straightforward in the absence of SET data. The first step involves a scan in energy of all the transistors on the chip using the laser to determine whether any of the SETs lie outside the area in $\Delta V-\Delta t$ phase space defined by the threshold values for propagation $\left(\Delta V_{\mathrm{th}}\right.$ and $\left.\Delta t_{\mathrm{th}}\right)$. If all the points lie inside the box, then one can accept the part together with the associated risk that the laser data did not uncover all SETs. Experiments performed to date indicate that the laser is highly reliable at reproducing the SETs produced by heavy ions, and have revealed no devices for which this approach is not successful. Therefore, such risk is expected to be small. Alternatively, one can perform an ion-beam test using only the highest LET ions available to confirm that all SETs are within the area of nontransmission in $\Delta V-\Delta t$ phase space. If some of the SETs are outside this window, a design engineer should be consulted to determine whether the follow-on circuit can be modified to increase $\Delta V_{\text {th }}$ and/or $\Delta t_{\text {th }}$ to prevent SET propagation. Such modifications may involve the addition of a filter, for example. Alternatively, if such changes are unacceptable to the mission, then it is necessary to perform a complete heavy-ion test.

\section{B. Heavy-Ion SET Data Available}

There are two possible options for the case where heavy-ion SET data are available. The first is when data are available for the configuration identical to the one of interest. No further testing is required, and it is a relatively straightforward decision to either accept the device as is, because it meets the requirement or, if it does not, to add a filter, if possible.

The other option - data are available for the device but in a different configuration-involves the following steps. The first step is the same as in Section III-A: a scan in energy of all the transistors using the pulsed laser to determine whether any SETs will propagate for the configuration of interest. If all the SETs have amplitudes and widths that are less than the threshold values for propagation, one can accept the part with the attendant risk (small), or perform a limited ion test using only the highest available LET ions. If some of the measured transients 


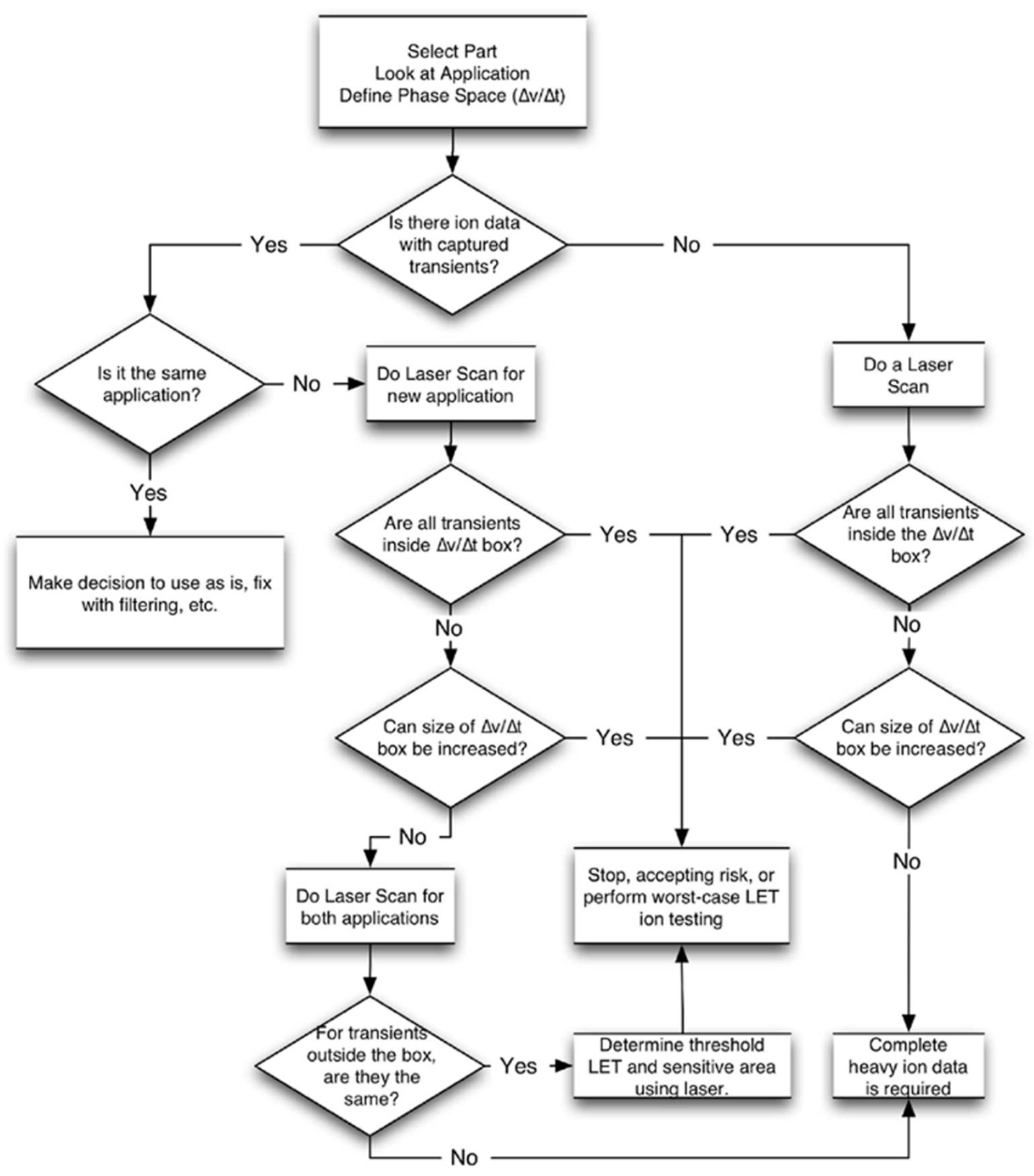

Fig. 3. Steps involved in using a pulsed laser for qualifying a part for SET response.

have amplitudes and widths greater than the thresholds for propagation, the design engineer should be consulted to determine whether the propagation requirements on $\Delta V_{\mathrm{th}}$ and/or $\Delta t_{\mathrm{th}}$ can be modified.

If the follow-on circuit cannot be modified to be tolerant of SETs, a scan of all the transistors using the pulsed laser should be performed for the same configuration as for the heavy-ion data. The laser and ion data for the original configuration are then combined in a single plot, which is compared with the one for the new configuration. If the loci of $(\Delta V, \Delta t)$ points are different for the two configurations, the laser cannot be used, and a complete heavy ion test must be done to fully characterize the part. However, if the same transistors in both configurations are responsible for the $(\Delta V, \Delta t)$ points located beyond the minimum values for propagation, the pulsed laser can be used to obtain a rough measure of LET threshold and cross-section for the new configuration.

The threshold is determined by calibrating the laser energy needed to produce an SET in the new configuration against that in the old configuration. This is done by placing the laser light on the transistor responsible for the $(\Delta V, \Delta t)$ points just outside the box in the old configuration and measuring the energy to produce that point. Then the device is placed in the new configuration and the energy measured at that same transistor to produce a $(\Delta V, \Delta t)$ point just outside the box. Since the ion LET threshold for the old configuration is known, it can be cal- 


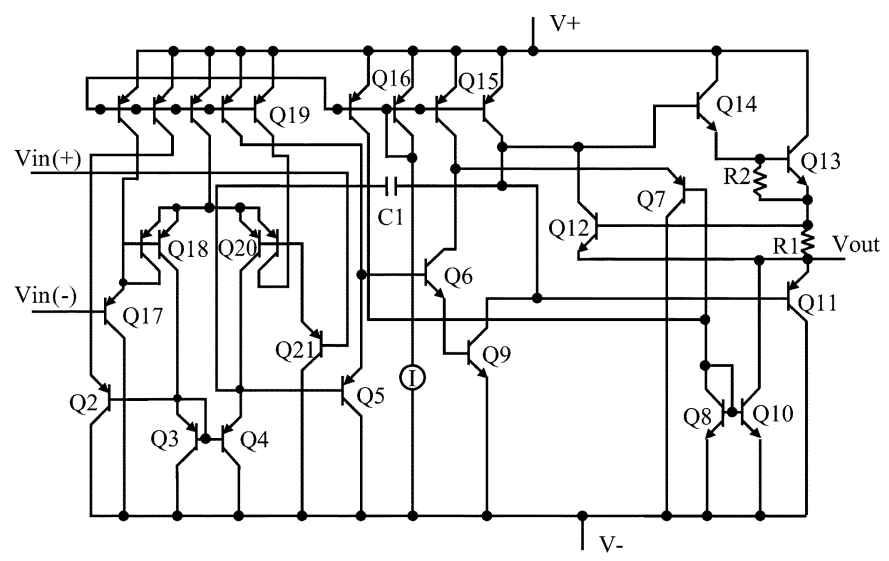

Fig. 4. Circuit schematic for the LM124 operational amplifier.

culated in the new configuration by taking the ratio of the laser energies and multiplying by $\mathrm{LET}_{\mathrm{th}}$ in the old configuration.

The saturated cross-section is easily measured by using the laser with high pulse energy to determine the sensitive area associated with every transistor that produces SETs that will propagate. Summing all those areas will give a measure of the saturated SET cross-section applicable to that particular application.

\section{EXAMPLES}

Two examples illustrating how to use the methodology are presented. They involve two different devices-an operational amplifier (LM124) that produces a wide variety of pulse shapes and a voltage comparator (LM111) that produces transients whose shapes are independent of which transistor is irradiated.

\section{A. $L M 124$}

SETs in the LM124 operational amplifier have been studied in considerable detail for a variety of different configurations. However, no detailed comparisons have been made of SET amplitudes and widths for different configurations.

The data shown in Fig. 1 were taken for the LM124 configured as a voltage follower with supply voltage of $\pm 15 \mathrm{~V}$ and an input of $5 \mathrm{~V}$. To illustrate the approach with a simple example, we make an arbitrary assumption that the LM124 is slated to be used in a system for which only negative transients longer than $20 \mu$ s are able to propagate in the follow-on circuitry. Inspection of Fig. 1 reveals that three transistors are capable of producing transients with widths greater than $20 \mu \mathrm{s}$. With reference to the circuit schematic of the LM124, shown in Fig. 4, together with knowledge of the circuit layout, the three transistors could be identified as Q9, Q16, and Q19, as is noted in Fig. 1. Q9 is located at the circuit output, whereas Q16 and Q19 are part of the current supply circuit.

Measurements were made of the laser energies required to produce transients in those three transistors. The relative amounts of energy required for producing SETs in transistors Q9, Q19, and Q16 were, respectively, 7:9:12. Therefore, the most sensitive transistor is Q9, and the least sensitive is Q16. Because the sensitive junctions of all three transistors are at the same depth, the relative laser pulse energies translate directly into relative LETs.

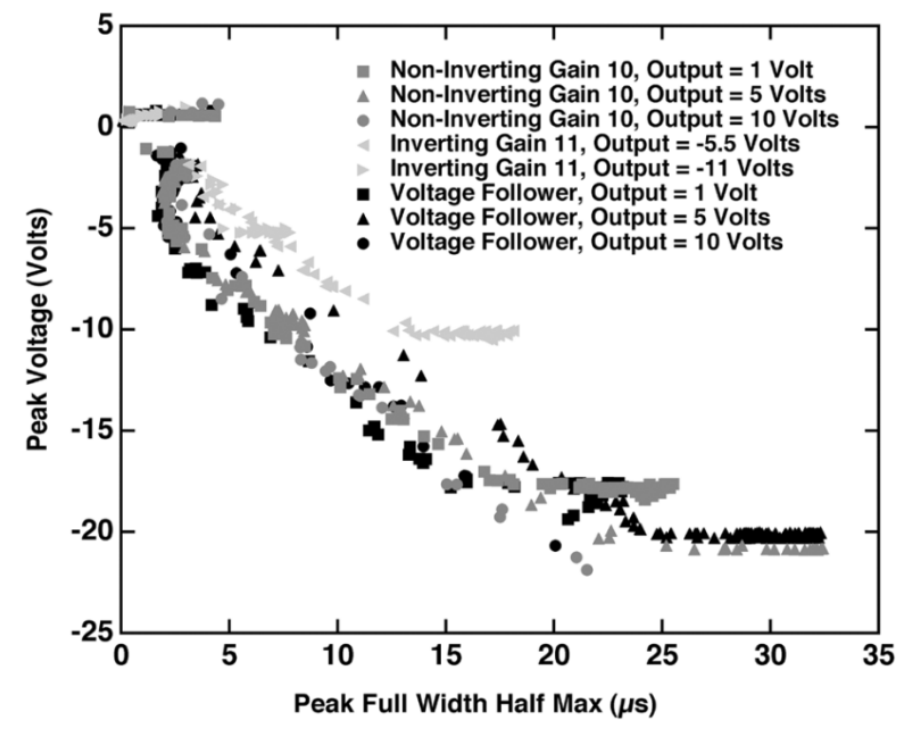

Fig. 5. Plot of laser-induced SET peak voltage versus width for a variety of input voltages and configurations. Each color corresponds to a different configuration.

To see whether changing the circuit conditions had any effect on the SET's shape or threshold, we focused the light on each transistor in the circuit and monitored the resulting SETs with an oscilloscope while varying the biases and configuration. A surprising result was that, for the most part, the transients did not change. That is, the SET amplitudes and widths remained constant as the supply voltage was varied from $\pm 10 \mathrm{~V}$ to $\pm 15 \mathrm{~V}$, the input voltage was varied from 1 to $5 \mathrm{~V}$, and the configuration was switched from voltage follower to inverting amplifier with gain of 11. The threshold levels were also measured to evaluate whether they were dependent on configuration. That involved adjusting the laser light intensity so that transient amplitudes were just above the oscilloscope's trigger level and monitoring them as the circuit conditions were changed. Again, for this particular device, no changes in SET amplitude or width were observed.

There is one parameter, however, that does affect the SETs-the voltage difference between the output and the rail. As the output voltage increases so does the voltage difference between the output and the negative rail. Larger amplitude SETs are thus possible. Fig. 5 shows the $\Delta V-\Delta t$ plot for SETs generated at transistor Q19 with pulsed laser light. The data clearly show that the saturated amplitudes (those falling along a horizontal line) increase as the input voltage increases. For example, when the output voltage is set to $5 \mathrm{~V}$, the distance to the negative rail is $20 \mathrm{~V}$ and the saturated values are indeed at $-20 \mathrm{~V}$. When the output is set to $-5.5 \mathrm{~V}$, the SETs have maximum amplitudes of $-9.5 \mathrm{~V}$.

The independence of the SET cross-section on applied voltages and device configuration are confirmed by the data in Fig. 6, which is a plot of SET cross-section versus ion LET for a number of different circuit conditions. The plot clearly shows that the LET threshold and saturated cross-section do not depend on circuit conditions, i.e., all the curves are coincident. Therefore, applied voltages and device configuration play very little role in determining SET rates for this device in space. 


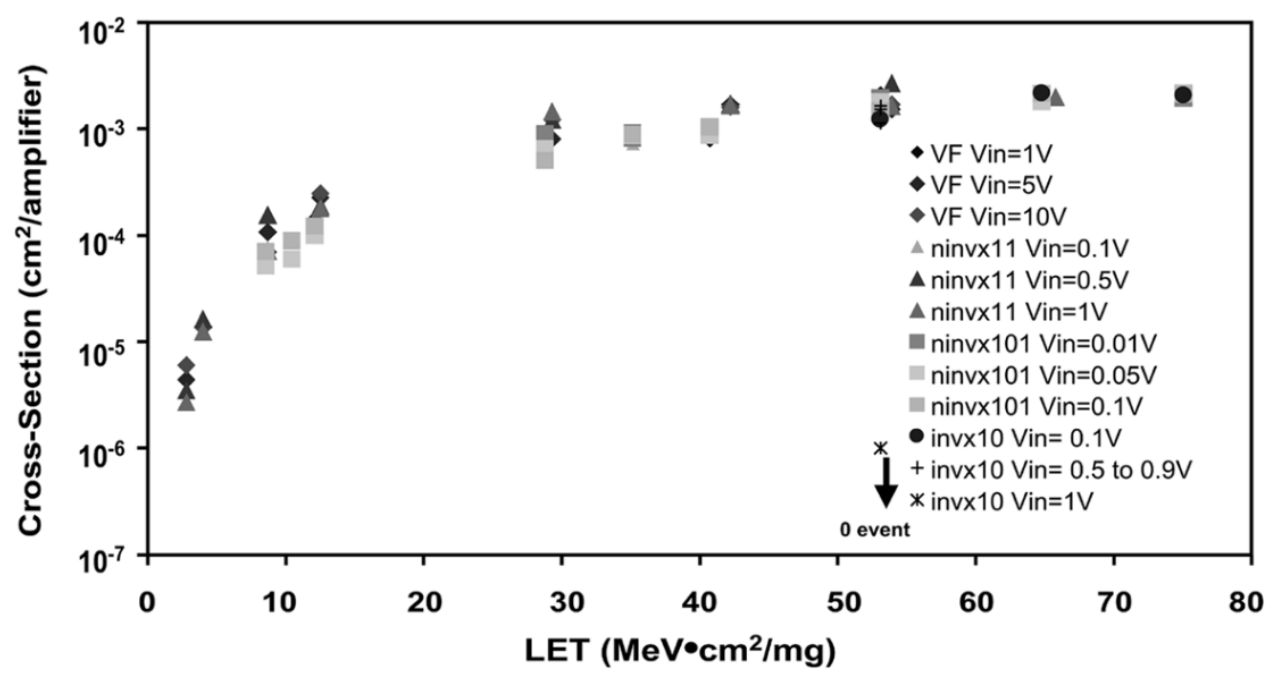

Fig. 6. Heavy-ion induced SET cross-section as a function of ion LET for the LM124 operational amplifier for a variety of different configurations. For all measurements, $V_{\mathrm{dd}}=+15 \mathrm{~V}$ and $V_{\mathrm{ss}}=-15 \mathrm{~V}$.

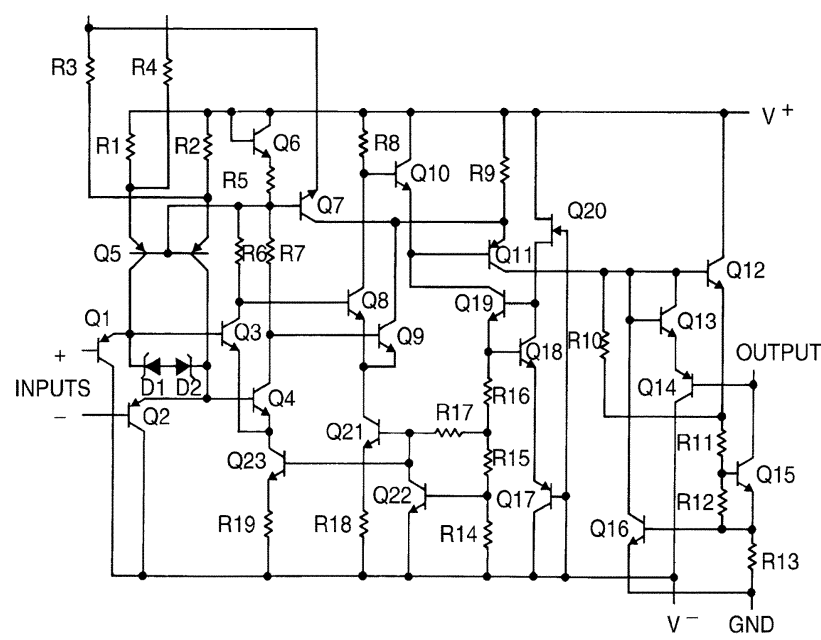

Fig. 7. Circuit diagram of the LM111.

These results indicate that one set of SETs obtained with heavy ions is sufficient to establish a base with which to compare quantitatively any other SET generated in the LM124.

\section{B. $L M 111$}

The LM111 was selected for testing because data clearly show that the SET cross-section depends critically on differential input voltage, particularly when the differential input voltage is small [3]. All the transients produced in the LM111 have the same shape, regardless of which transistor is the source. With the output of the LM111 connected via a $1.7-\mathrm{k} \Omega$ pull-up resistor to $+15 \mathrm{~V}$ and the positive input set at $0.1 \mathrm{~V}$ above the negative input, all the SETs are negative. The pulsed laser was used to scan the chip to identify the sensitive transistors. Seven transistors and one diode were determined to be SET-sensitive. They are Q1, D1, Q58, Q4, Q8, Q11, Q12, and Q15 (shown in Fig. 7). Only those SETs generated at Q2 and D1 exhibit any sensitivity to differential input voltage. Therefore, in comparing the SET sensitivity of the LM111 for two different input voltages, only SETs generated at Q2 and D1 need be compared.

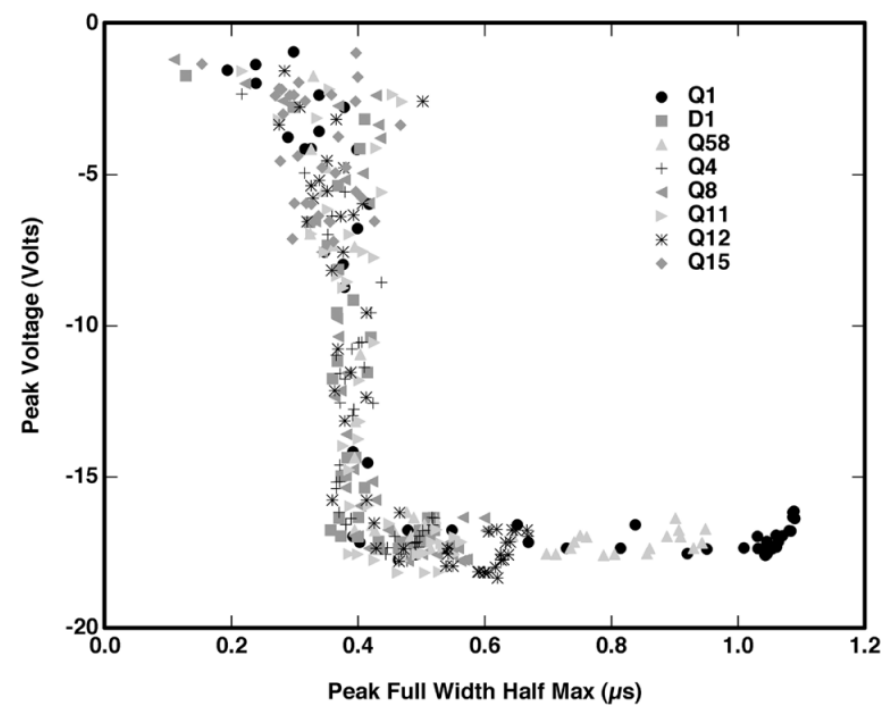

Fig. 8. Plot of laser-induced SET peak voltage versus width for seven transistors and one diode on the LM111. All the transients have the same shape but different sensitivity. The most sensitive transistor is Q1 and it produces the longest SETs. $V_{\mathrm{dd}}=15 \mathrm{~V}, V_{\mathrm{ss}}=-15 \mathrm{~V}, \Delta V_{\text {in }}=0.1 \mathrm{~V}$ and the resistance of the pull-up resistor is $1.7 \mathrm{k} \Omega$.

Fig. 8 shows a plot of amplitude versus width for all the SETs observed in the LM111 for a differential input voltage of $0.1 \mathrm{~V}$. The data show that the shapes of all the SETs are identical, no matter their origin. The most sensitive transistor, Q1, produces the largest SETs with maximum amplitudes of $-18 \mathrm{~V}$ and maximum widths of just over $1 \mu \mathrm{s}$. It is a simple exercise to decide whether SETs generated in the LM111 will propagate through follow-on circuitry having a known bandwidth. For example, for the LM111 configured the same as for Fig. 8, there are no transients longer than $1.2 \mu \mathrm{s}$.

We note that the shape of the SETs will change if the voltage across the pull-up resistor or the value of the resistance of the pull-up resistor is changed. Increasing both the voltage across the resistor and the value of the resistance will affect the locus of the $(\Delta V, \Delta t)$ points in Fig. 8. The points will cover greater distances along both the $x$ and $y$ axes. 
The LM111 contains one transistor and one diode whose SET sensitivities depend critically on differential input voltage. As the differential input voltage increases, the sensitivities decrease, and for sufficiently high voltages the transistor and diode are not SET sensitive. All the other SET-sensitive transistors are independent of differential input voltage.

The information obtained with the laser, such as the independence of the SET sensitivities of most of the transistors to differential input voltage, can be used to fully characterize the part for any application requiring a different input voltage using a single accelerator test. The only change that occurs with input voltage is to the SET sensitivities of the input transistor and diode. Relative measurements of their SET thresholds as a function of $V_{\text {in }}$ using a pulsed laser can be used to determine the LET thresholds by multiplying the LET threshold measured with ions by the ratio of the laser pulse energies for the two configurations.

If the LM111 is to be used in a configuration in which the output impedance is different, the shapes of the transients will change. Plots of $\Delta V$ versus $\Delta t$ obtained with a laser may be used together with information about the bandwidth of the follow-on circuit in the intended application to assess whether any transients will propagate.

\section{Summary AND CONCLUSION}

We have proposed a methodology for using pulsed laser light to reduce the amount of heavy-ion testing needed to qualify linear circuits for space missions. The methodology is based on the excellent agreement observed between single event transients produced by the two ionization sources. The approach is based on analyzing plots of amplitude $(\Delta V)$ versus width $(\Delta t)$ for SETs generated by pulsed laser light and heavy ions, taking into account the unique mission-specific requirements for individual applications. The use of the laser introduces flexibility and robustness into the mission qualification process, and can significantly reduce the amount of heavy ion testing required. The utility of this approach originates in the ability of the pulsed laser to reproduce accurately the complete spectrum of transients produced by heavy ion irradiation in the wide range of linear devices that have been investigated to date. In addition, the incorporation of pulsed laser measurements in the validation process flow provides (directly) node-specific information that cannot be extracted from broad-beam heavy-ion measurements. Such value-added information permits the design engineer to make better-informed decisions regarding part acceptance or rejection.

The pulsed laser evaluation plays several different roles within the proposed methodology. In one case, in which heavy ion data is available for a configuration different from the one of interest, the laser results can be used to correlate the SET behavior of the device in the different configurations. Based on such results, a decision can be made as to whether the available data sets are sufficient, or whether additional ion tests are required. In another scenario, in which no heavy-ion data is available, pulsed laser SET measurements can be used to quickly determine the worst-case transients generated by each node of the circuit. Such information can be used together with the mission-specific requirements for that part to determine part acceptance, or whether heavy ion testing is warranted. Part acceptance, in this case, requires an educated decision by the design engineer, and acceptance of the risk that a potentially harmful node was not uncovered in the laser measurements (because of extreme metal coverage, for example). In those cases where additional ion testing is required, the combination with laser measurements often minimizes the number of necessary ion measurements, sometimes to a single LET value.

Finally, if there is doubt regarding the ability of the pulsed laser to produce SETs for a particular device or configuration, such as when a transistor is completely covered with metal, or when different transistors are SET-sensitive in different configurations, the conservative approach involving heavy-ion testing should be taken.

\section{REFERENCES}

[1] R. Koga, S. D. Pinkerton, S. C. Moss, D. C. Meyer, S. LaLumondiere, S. J. Hansel, K. B. Crawford, and W. R. Crain, "Observation of single event upsets in analog microcircuits," IEEE Trans. Nucl. Sci., vol. 40, pp. 1838-1844, Dec. 1993.

[2] R. Ecoffet, S. Duzellier, P. Tastet, C. Aicardi, and M. Labrunee, "Observation of heavy-ion induced transients in linear circuits," in 1994 Radiation Effects Data Workshop Record, July 1994, pp. 72-77.

[3] R. Koga, S. H. Penzin, K. B. Crawford, W. R. Crain, S. C. Moss, S. D. Pinkerton, S. D. LaLumondiere, and M. C. Maher, "Single event upset (SEU) sensitivity dependence of linear integrated circuits (ICs) on bias conditions," IEEE Trans. Nucl. Sci., vol. 44, pp. 2325-2332, Dec. 1997.

[4] D. Lewis, V. Pouget, F. Beaudouin, P. Perdu, H. Lapuyade, P. Fouillat, and A. Touboul, "Backside laser testing of ICs for SET sensitivity evaluation," IEEE Trans. Nucl. Sci., vol. 48, pp. 2193-2201, Dec. 2001.

[5] R. Koga, S. H. Crane, K. B. Crawford, S. C. Moss, S. D. LaLumondiere, and J. W. Howard, "Single event transient (SET) sensitivity of radiation hardened and COTS voltage comparators," in 2000 IEEE Radiation Effects Data Workshop, July 2000, pp. 53-60.

[6] F. Miller, A. Germain, N. Buard, R. Gaillard, P. Poirot, C. Chatry, T. Carriere, and R. Dufayel, "Interest of laser test facility for the assessment of natural radiation environment effects on integrated circuits based systems," presented at the RADECS 2003 Conf., Noordwijk, Holland, Sept. 2003.

[7] R. Pease, A. Sternberg, Y. Boulghassoul, L. Massengill, S. Buchner, D. McMorrow, D. S. Walsh, G. L. Hash, S. LaLumondiere, and S. Moss, "Comparison of SETs in bipolar linear circuits generated with an ion microbeam, laser light, and circuit simulation," IEEE Trans. Nucl. Sci., vol. 49, pp. 3163-3170, Dec. 2002.

[8] S. Buchner, D. McMorrow, A. Sternberg, L. Massengill, R. L. Pease, and M. Maher, "Single event transient (SET) characterization of an LM119 voltage comparator: An approach to SET model validation using a pulsed laser," IEEE Trans. Nucl. Sci., vol. 49, pp. 1502-1508, June 2002.

[9] D. McMorrow, W. T. Lotshaw, J. S. Melinger, S. Buchner, and R. L. Pease, "Subbandgap laser-induced single event effects: Carrier generation via two-photon absorption," IEEE Trans. Nucl. Sci., vol. 49, pp. 3002-3008, Dec. 2002.

[10] P. Adell, R. D. Schrimpf, J. J. Barnaby, R. Marec, C. Chatry, P. Calvel, C. Barillot, and O. Mion, "Analysis of single-event transients in analog circuits,” IEEE Trans. Nucl. Sci., vol. 47, pp. 2616-2623, Dec. 2000.

[11] R. Marec, C. Chatry, P. Adell, O. Mion, C. Barillot, P. Calvel, and L. Cresciucci, "Toward a single event transient hardness assurance methodology," presented at the RADECS 2001 Conf., Grenoble, France, Sept. 2001.

[12] S. Buchner, D. McMorrow, C. Poivey, J. Howard, R. L. Pease, M. Savage, L. Massengill, and Y. Boulghassoul, "Comparison of single-event transients induced in an operational amplifier (LM124) by pulsed laser light and a broad beam of heavy ions," IEEE Trans. Nucl. Sci., vol. 51, pp. 2776-2781, June 2004. 